\title{
Numerical and experimental investigation on the prevention of CO deflagration
}

\author{
Yu Zhang*, Lixin Yu, Xiaolin Wei, Teng Li, Bo Li \\ Institute of Mechanics, Chinese Academy of Sciences, Beisihuanxi Road 15, Beijing, China
}

\section{A R T I C L E I N F O}

\section{Article history:}

Received 24 July 2008

Received in revised form

17 November 2008

Accepted 21 November 2008

\section{Keywords:}

$\mathrm{CO}$

Deflagration

Preventing deflagration

\begin{abstract}
A B S T R A C T
The exhaust gases from industrial furnaces contain a huge amount of heat and chemical enthalpy. However, it is hard to recover this energy since exhaust gases invariably contain combustible components such as carbon monoxide (CO). If the $\mathrm{CO}$ is unexpectedly ignited during the heat recovery process, deflagration or even detonation could occur, with serious consequences such as complete destruction of the equipment. In order to safely utilize the heat energy contained in exhaust gas, danger of its explosion must be fully avoided. The mechanism of gas deflagration and its prevention must therefore be studied. In this paper, we describe a numerical and experimental investigation of the deflagration process in a semi-opened tube. The results show that, upon ignition, a low-pressure wave initially spreads within the tube and then deflagration begins. For the purpose of preventing deflagration, an appropriate amount of nitrogen was injected into the tube at a fixed position. Both simulation and experimental results have shown that the injection of inert gas can successfully interrupt the deflagration process. The peak value of the deflagration pressure can thereby be reduced by around $50 \%$.
\end{abstract}

(c) 2008 Elsevier Ltd. All rights reserved.

\section{Introduction}

The shortage of energy is currently one of the most serious problems facing the whole world. On the other hand, the huge amount of heat and chemical enthalpy contained in the exhaust gases from industrial furnaces is being lost every day. For example, considering only the converter gas used in metallurgical processing, some 60 thousand million cubic meters of exhaust coal gas are burned in vain every year in China. Besides wasting energy, the emission of high-temperature and carbon-containing gas is also environmentally damaging.

The converter gas contains up to $60 \% \mathrm{CO}$ and its initial venting temperature could reach $1450^{\circ} \mathrm{C}$. In order to recover the sensible heat value of the exhaust gas an additional postheat exchanger should be furnished. When gas flows though the device it will be cooled down and meanwhile a proper amount of steam will be produced from the heat exchanger for the electricity generation. The cooled mixture still contains combustible components then it can be used as fuel gas.

However, because the gas always contains combustible components ( $\mathrm{CO}$ ), the heat value of exhaust gas is dangerous to recover. If the $\mathrm{CO}$ is unexpectedly ignited during the heat recovery

\footnotetext{
* Corresponding author. Tel.: +8610 82544231; fax: +8610 62561284

E-mail address: zhangyu@imech.ac.cn (Y. Zhang).
}

process, damaging deflagration could occur and the equipment could be completely destroyed. To safely utilize the recoverable thermal energy from exhaust gas, the potential danger of $\mathrm{CO}$ explosion must be rigorously avoided. The CO deflagration process must therefore be studied in detail with a view to preventing explosion.

In the sequent discussions the initial CO mass component in the mixture will be kept as $23 \%$. Under this condition CO will be most likely to be ignited and the pressure peak value due to deflagration will be close to the highest. If the CO deflagration could still be quenched under such serious condition then the proposed preventing method in this paper would be validated for broader usages.

The physical nature of deflagration may be attributed to the formation and development of a premixed flame. In 1926, Chapman and Wheeler were the first to investigate the spreading of a flame in a tube with internal obstacles (Glassman, 1996). Moen (Chan, Moen, \& Lee, 1983; Moen, Donato, \& Knystautas, 1980) experimentally investigated the acceleration of a deflagration flame. Chan analyzed the effect of a rectangular obstacle on the flame speed (Lee, Knystautas, \& Freiman, 1984). Lee explored the mechanism of the deflagration-to-detonation transition (Lee, Knystautas, \& Chan, 1984; Patel, Ibrahim, Yehia, \& Hargrave, 2003; Teodorczyk, Lee, \& Knystautas, 1988). Yu experimentally tested the effect of internal obstacles on flame acceleration and the development of an overpressure (Yu, Sun, \& Wu, 2002a, 2002b). However, previous research work has been mainly focused on the deflagration of 


\begin{tabular}{|c|c|}
\hline \multicolumn{2}{|c|}{ Nomenclature } \\
\hline$R_{i}$ & $\begin{array}{l}\text { Volumetric chemical reaction rate of component } i \\
\left(\mathrm{~kg} / \mathrm{m}^{3} \mathrm{~s}\right)\end{array}$ \\
\hline$\rho$ & Density $\left(\mathrm{kg} / \mathrm{m}^{3}\right)$ \\
\hline$\xi^{*}$ & Length scale of the turbulence fine structures (m) \\
\hline$\tau^{*}$ & Time scale of the turbulence fine structures (s) \\
\hline$Y^{*}$ & $\begin{array}{l}\text { Concentration of component } i \text { in fine structures }(\mathrm{kg} / \\
\mathrm{kg})\end{array}$ \\
\hline$Y_{i}$ & $\begin{array}{l}\text { Concentration of component } i \text { in fine structures } \\
\text { after } \tau^{*}(\mathrm{~kg} / \mathrm{kg})\end{array}$ \\
\hline$\nu$ & Kinetic viscosity $\left(\mathrm{m}^{2} / \mathrm{s}\right)$ \\
\hline$\varepsilon$ & $\begin{array}{l}\text { Dissipation rate of the turbulence kinetic energy } \\
(\mathrm{W} / \mathrm{s})\end{array}$ \\
\hline$k$ & Turbulence kinetic energy $(\mathrm{W})$ \\
\hline
\end{tabular}

hydrocarbon gases. For pure, dry CO, which is rather difficult to ignite, reported experimental work is very rare.

In this paper, we describe a numerical and experimental investigation of the process of $\mathrm{CO}$ deflagration in a semi-opened tube. Both the K-epsilon (K-E) and Reynolds stress (R-S) turbulence models, coupled with the same EDC (eddy dissipation concept) combustion model, have been used to simulate the deflagration process. It has been found that the results predicted by the R-S model more closely resemble those found experimentally because the K-E model always overestimates the turbulence for anisotropic turbulent flows. Based on the results of the simulation, it can be seen that the deflagration happens only a little while after the pressure is disturbed due to ignition leaving the spark point. An appropriate amount of nitrogen was injected into the semi-opened tube as the deflagration started. Both experimental and simulation results have shown that, by injecting nitrogen in this way, the deflagration can be successfully interrupted. The pressure peak value is consequently greatly reduced.

\section{CFD modeling}

There are two factors that strongly affect the deflagration process. One is the turbulence of the fluid and the other is the chemical kinetics of the reactions. Both the K-E and R-S models were used independently to describe the turbulence distribution in the reactor. A standard EDC model was used to calculate the volumetric chemical reaction rate, which is described by Eqs. (1)-(3). In the EDC model, there are two important parameters that determine the calculated reaction rate, the so-called length scale and the time scale of the turbulence fine structures. Both of these parameters are mainly dependent on the turbulence kinetic energy. Therefore, the final predicted chemical reaction rates are not only determined by the EDC model, but are also dependent on the turbulence model. Full details of the models mentioned above can be found in Fluent 6.3 documentation.

$$
\begin{aligned}
& R_{i}=\frac{\rho\left(\xi^{*}\right)^{2}}{\tau^{*}\left[1-\left(\xi^{*}\right)^{3}\right]}\left(Y_{i}^{*}-Y_{i}\right) \\
& \tau^{*}=C_{T}\left(\frac{v}{\varepsilon}\right)^{1 / 2} \\
& \xi^{*}=C_{\xi}\left(\frac{v \varepsilon}{k^{2}}\right)^{3 / 4}
\end{aligned}
$$

Fig. 1 displays the whole calculation domain. Zone number 1 represents the semi-opened tube in which the deflagration occurs. Zone number 2 represents the open space that is attached to the semi-opened tube. All boundary conditions can be found in Fig. 1. Initially, $\mathrm{CO}$ and air are thoroughly mixed and introduced into the semi-opened tube. A spark is then created at the center of the left wall. The mixture is ignited and deflagration begins. When the pressure signal due to the ignition reaches the tube outlet, an appropriate amount of nitrogen is injected into the tube to stop the deflagration.

The simulation is two dimensional. A set of rectangle grids is applied. The total grid number is 7087. The maximum face area is $2.74 \times 10^{-3} \mathrm{~m}^{2}$ and the minimum face area is $4.23 \&$ times; $10^{-5} \mathrm{~m}^{2}$. The case is unsteady and the time step is set up as $10^{-5} \mathrm{~s}$. The convergence criteria for all conservation equations are defined as the residual of which is less than $10^{-5}$. All walls are set up as smooth wall which means no wall roughness was taken into account for the simulations.

\section{Experimental}

Fig. 2 shows a schematic representation of the experimental system. It consists of three parts: a gas supply section, a test section, and the deflagration prevention section. Initially, air (1) and $\mathrm{CO}$ (4) flow together into the electrical heater (6). It should be pointed out that the temperatures of exhaust gases from real furnaces are always much higher than room temperature. Therefore, the electrical heater is incorporated here to heat the mixed $\mathrm{CO}$ and air. However, in later discussions the heater is not considered because the effect of temperature on the deflagration is beyond the scope of this paper. The test section is a semiopened tube as depicted in Fig. 1. It is divided into two parts, a clear tube section (7) and a further tube section with internal adjacent annular obstacles (8). Furthermore, eight highly sensitive pressure detectors are arranged along the test section of the tube. Table 1 gives the precise positions of all of the detectors. Detectors

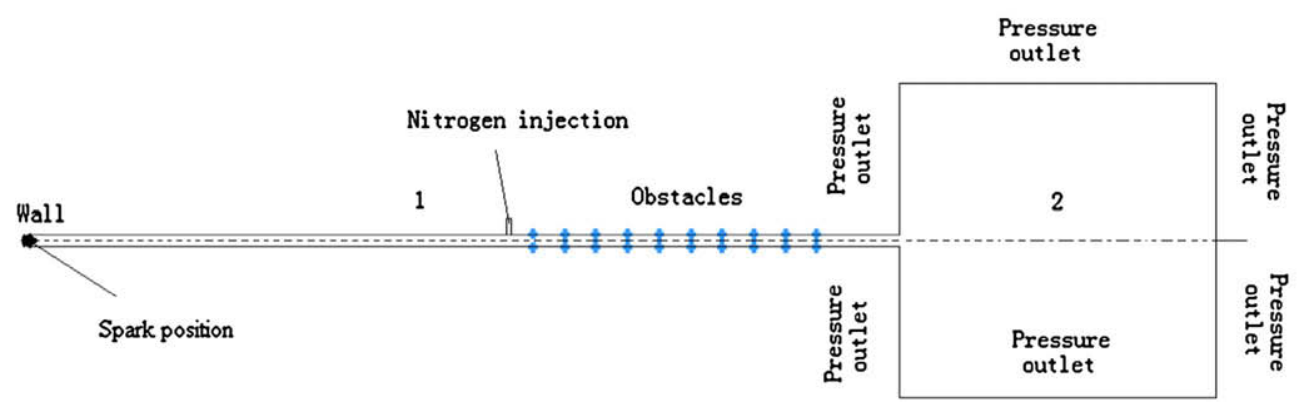

Fig. 1. Scheme of the simulation domain. 


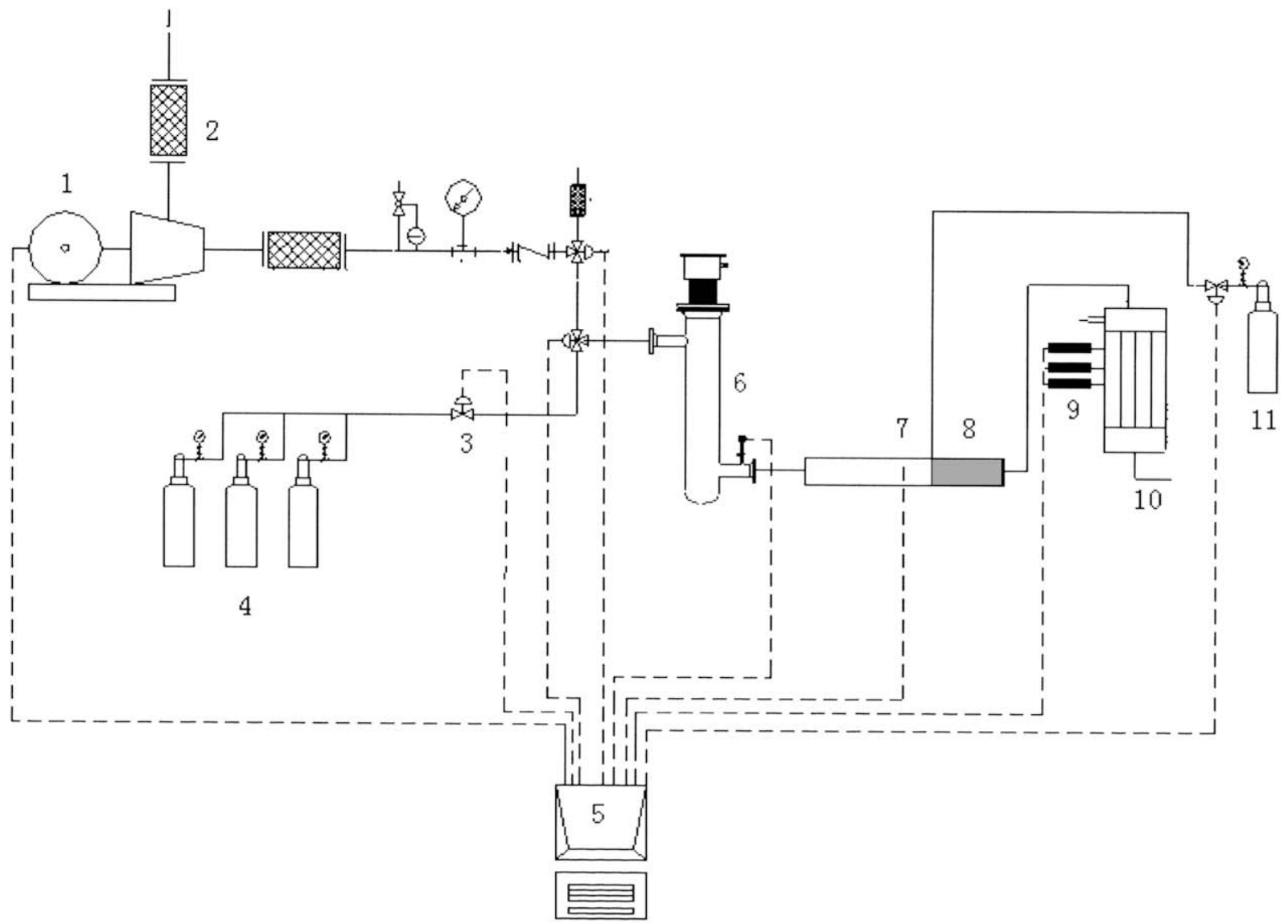

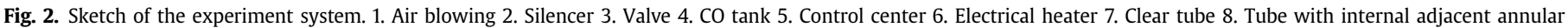
obstacles. 9. Pressure detector. 10. Water cooler. 11. Nitrogen tank.

1-4 are positioned in the clear part of the tube, while detectors 5-8 are positioned in the obstructed part. The whole test section is set up horizontally. The clear tube part is $4.0 \mathrm{~m}$ long while the obstructed tube part is $2.0 \mathrm{~m}$ long. The radius of clear tube part is $0.04 \mathrm{~m}$. The obstructed tube has the same radius and the blockage ratio inside is 0.375 . The CO mass component in the mixture is kept as $23 \%$. Initially the test section is full of such mixture gas with the room temperature $(290 \mathrm{~K})$ and one atmosphere pressure. During deflagration, the changing pressure at the different positions can be recorded in detail by these detectors. After the test, the exhaust gas is passed through a water cooler (10) and is then released to the atmosphere. In deflagration prevention tests, when the pressure signal due to the ignition is detected by the pressure detector (9), the control center (5) will order the nitrogen tank (11) to inject nitrogen at the connected positions of (7) and (8). The deflagration will thereby be interrupted. Fig. 3 shows a photograph of the actual arrangement of the experimental

Table 1

Positions of all pressure detectors.

\begin{tabular}{lll}
\hline $\begin{array}{l}\text { Detector } \\
\text { number }\end{array}$ & $\begin{array}{l}\text { Distance from the } \\
\text { left wall }(\mathrm{m})\end{array}$ & Location \\
\hline 1 & 0.5 & Clear section \\
2 & 1.5 & of the tube \\
3 & 2.5 & \\
4 & 4.0 & Obstructed section \\
5 & 4.5 & of the tube \\
6 & 5.0 & \\
7 & 5.5 & \\
8 & 6.0 & \\
\hline
\end{tabular}

instrumentation. It was taken from the perspective of the outlet of the test tube. The test tube, water cooler, nitrogen tank, and nitrogen injector can clearly be identified.

The obstructed tube is set up here to represent the heat exchanger and the clear tube represents the channel where the mixture gas flows through to the heat exchanger. The ignition sparks could be found in both channel and heat exchanger. And the most common case is, the mixture got ignited in the channel but deflagration happens in the heat exchanger because tube bank inside the heat exchanger can play the role like obstacles to accelerate the flame. Therefore the nitrogen injector is placed at the connection point of clear tube and obstructed tube for preventing the deflagration. The proper injection pressure of nitrogen is very much depended on the gas components. Various injection

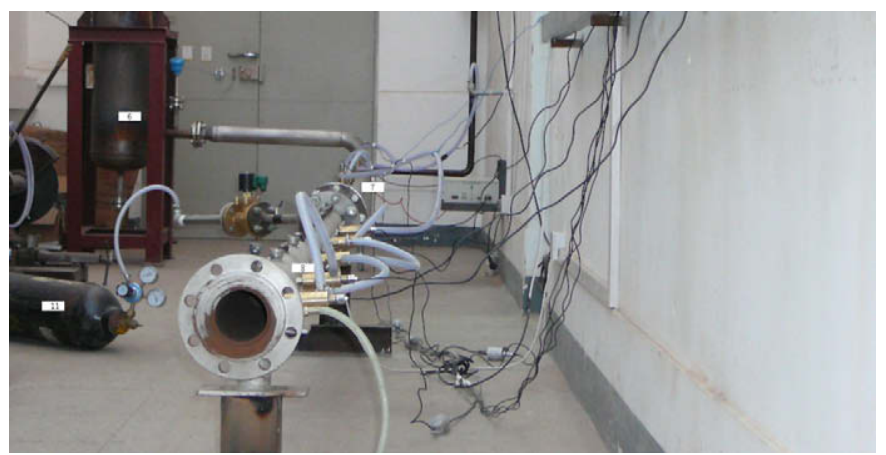

Fig. 3. Real picture of the experimental system. 
pressures have been tested and only the nitrogen of 0.4 MPa can totally quench the CO flame.

\section{Results and discussion}

\subsection{Ignition signal}

The ignition device is an electrical spark plug which is widely used in general kinds of vehicles. The energy released from it is around $10 \mathrm{~mJ}$ per spark. And the spark time is around $1 \mathrm{~ms}$.

Fig. 4 depicts the spreading process of the pressure disturbed by the ignition. Fig. 4-1 displays the simulation results. Initially, when ignition takes place, a pressure signal appears immediately at the left wall, attaining a value of $0.02 \mathrm{MPa}$. With the passing of time, the pressure wave flows from the left wall to the outlet. The highpressure region becomes broader, while its peak value remains almost the same. This means that the ignition energy is continuously converted into pressure potential energy. After $0.016 \mathrm{~s}$, the wave reaches the outlet and the pressure of all regions behind the wave is higher than the initial pressure (0 MPa gauge pressure). A high-pressure will greatly accelerate the chemical reaction, whereupon deflagration takes place. Fig. 4-2 shows the measured pressure signals due to ignition. From Fig. 4-2, it can be seen that the disturbed pressure value reaches $0.03 \mathrm{MPa}$. It can also be seen that the time sequence of the pressure peak value recorded by all of the pressure detectors corresponds to their sequential positions from the left wall to the outlet. The results show that the ignition is responsible for inducing the disturbed pressure. The pressure wave spreads inside the tube until deflagration takes place.

From the simulation results it can be seen that the ignition period is around $0.016 \mathrm{~s}$. However, from the experimental results the ignition period can be read as $0.06 \mathrm{~s}(0.14 \mathrm{~s}-0.08 \mathrm{~s}$ from Fig. $4-2)$. It is hard to tell which one is right because both simulation and
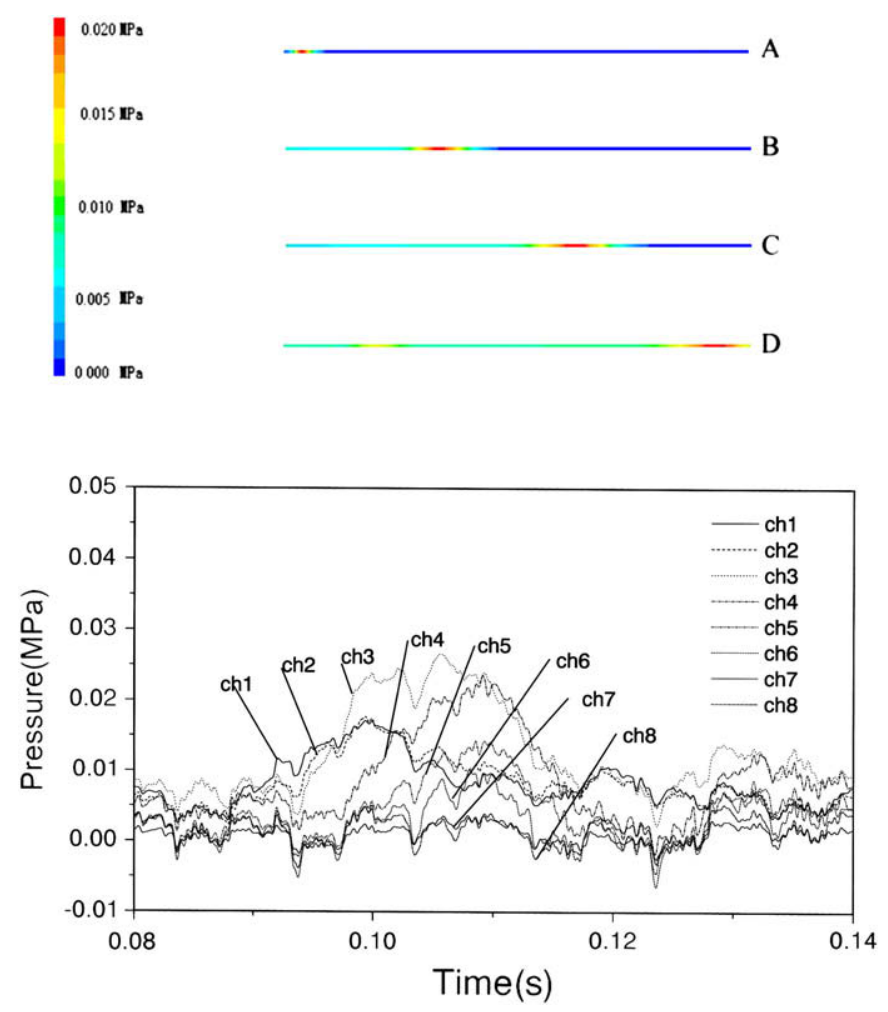

Fig. 4. The spread of pressure disturbed inside the tube after ignition. 4-1. Calculated results. A. $t=0.001 \mathrm{~s}$; B. $t=0.006 \mathrm{~s}$; C. $t=0.011 \mathrm{~s}$; D. $t=0.016 \mathrm{~s}$. $4-2$. Experimental results. measurement are not perfectly accurate. However it still can be concluded that the ignition period should be around 0.01-0.06 s. And it indicates that the optimal extinguishing moment should be no later than $0.06 \mathrm{~s}$ after ignition.

\subsection{Pressure increases when the flame travels}

Fig. 5 gives the predicted pressure history when the flame travels in both clear tube and obstructed tube. It shows that in clear tube part the pressure peak value is just $0.42 \mathrm{MPa}$ while in the obstructed tube part the maximum pressure can reach $0.72 \mathrm{MPa}$. This is because obstacles placed inside the tube can greatly accelerate $\mathrm{CO}$ flame and then the pressure peak value due to deflagration will be increased also. It still can be identified that, the starting time sequence of pressure increasing corresponds to the pressure detectors' sequential positions from the left wall to the outlet. However the time sequence of pressure peak value appearing at all detectors does not correspond to their sequential positions because once the pressure wave formed it does not spread just in the direction from left to right.

\subsection{Turbulence}

Fig. 6 shows the pressure peak at the 6 th detector. It should be pointed out that the original time in this figure is taken to be the start of the pressure peak rather than the start of the ignition. In
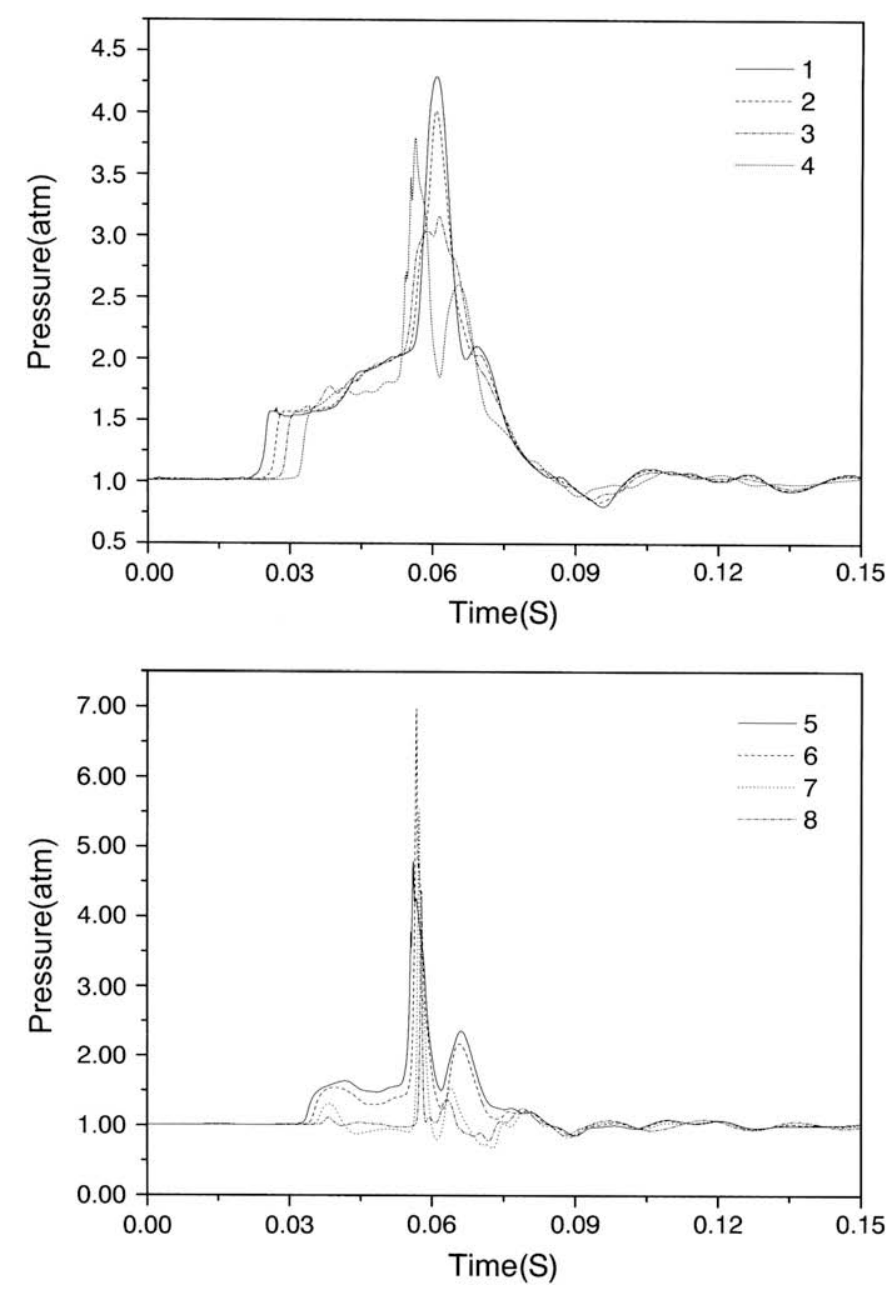

Fig. 5. The pressure increases when the flame travels and accelerates in the region with and without obstacles. (a) Without obstacles (b) With obstacles. 


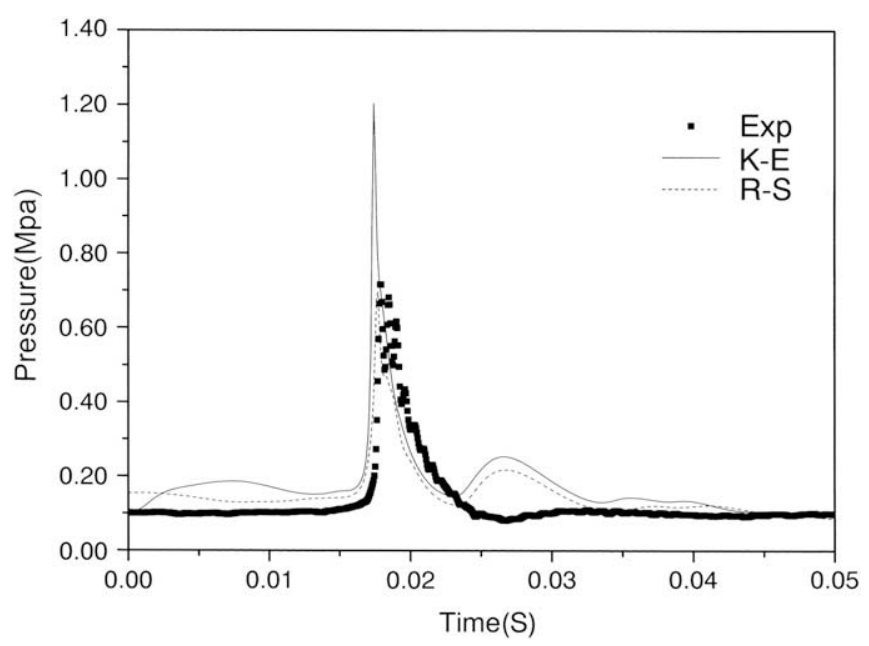

Fig. 6. The predicted and measured pressure peak at 6th position.

CFD simulation it is easily to make the recording time and the ignition time exactly same. However when doing experimental test the recording time should be always earlier than the ignition time for no important signal would be missed. Furthermore, for the operation convenience the recording device is always kept on until all of the expected tests got finished. Therefore when comparing the simulation results with the experimental results it has to make a new original time point for both. For this discussion, the original time point is defined as when the pressure at detector 6 is just going up but not the ignition time.

From Fig. 6, it can be seen that the lifetime of the pressure peak is around $0.01 \mathrm{~s}$. The solid line represents the predicted pressure using the K-E model and the dotted line represents the predicted pressure using the R-S model. Compared with the measured pressure, it can be seen that the R-S model gives a more reasonable estimate than the K-E model. This is because the K-E model invariably overestimates the turbulence for an anisotropic turbulent flow. Turbulence is one of the most important factors determining the chemical reaction rate. Therefore, overestimating the turbulence will result in an overestimation of the chemical reaction rate. This, in turn, will lead to an overestimation of the pressure induced by the reaction. The results imply that turbulence plays a very important role during deflagration, and so it may be possible to control deflagration by adjusting the turbulence.

As to the subject of this research, turbulence has two effects on the deflagration. First, when the mixture got ignited high turbulence will greatly accelerate the CO flame and then deflagration happens. Second, high turbulence will greatly enhance the heat transfer in the exchanger and then the combustible mixture will be cooled down more quickly and therefore the deflagration will be prevented. So it is important to do further research about the structures inside the heat exchanger for the purpose of getting higher heat transfer efficiency and lower incidence of deflagration happening.

\subsection{Extinguishing of deflagration}

Fig. 7 displays the pressure history at different positions when deflagration fully occurred. Pressure detector 2 is located in the clear part of the tube, while pressure detector 6 is positioned in the obstructed part. From both the experimental and simulation results, it can be identified that the highest pressure peak value appears at the position of the 6th pressure detector. It is located almost at the center of the obstructed part of the tube. And the
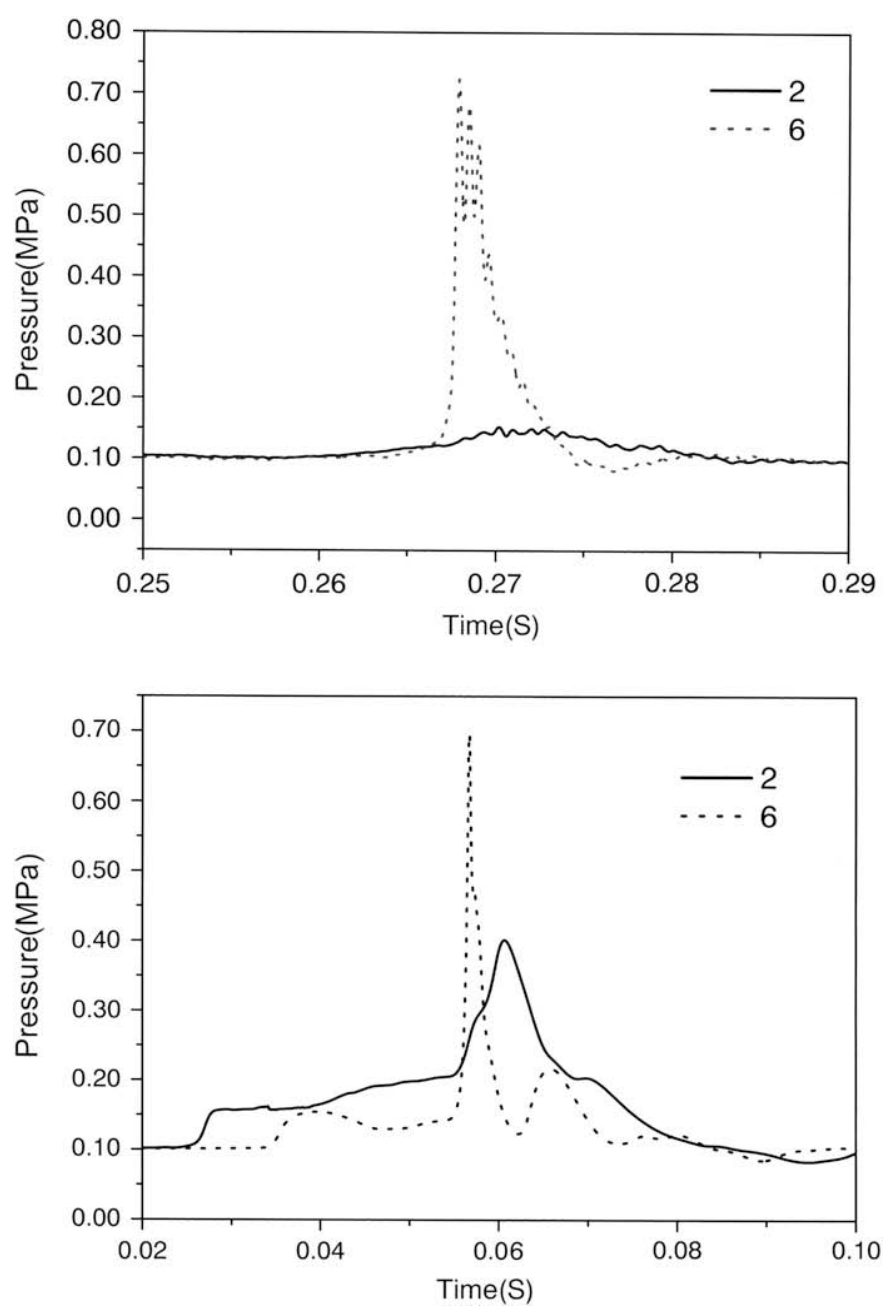

Fig. 7. The pressure at different positions during deflagration. 7-1. Experimental results. 7-2. Simulation results.

exact peak value captured by the detector is $0.72 \mathrm{MPa}$. For comparison, the estimated maximum pressure in a closed and rigid vessel using traditional thermodynamic method has been given below:

$P_{\max }=P_{\text {start }} \times\left(\frac{T_{\max }}{T_{\text {start }}} \frac{\sum n_{2}}{\sum n_{1}}\right)$

The maximum temperature in that vessel is assumed to be $2500 \mathrm{~K}$ which is as same as the temperature peak value got from CFD simulation. And at time beginning the vessel is full of the same mixture as in the test tube. The calculated final pressure in the vessel is $0.78 \mathrm{MPa}$ which is close to the CFD predicted pressure peak value. The results imply that when pressure peak appears in the tube there is almost no gas expanding at the flame surface.

Fig. 7-1 also shows the peak value at the 2nd pressure detector. It appears later than the moment when the 6th detector registers the pressure peak. This is because when the pressure peak appears at the 6th detector, the pressure wave will spread both upstream and downstream. When it reaches the upstream position, where detector number 2 is located, the pressure signal will be registered. Consequently, the pressure peak time for the 2 nd detector is later than that for the 6th detector. Moreover, the pressure will be weakened during its spread because of the friction of the wall. Therefore, the peak value at the 2 nd detector is much smaller. The 


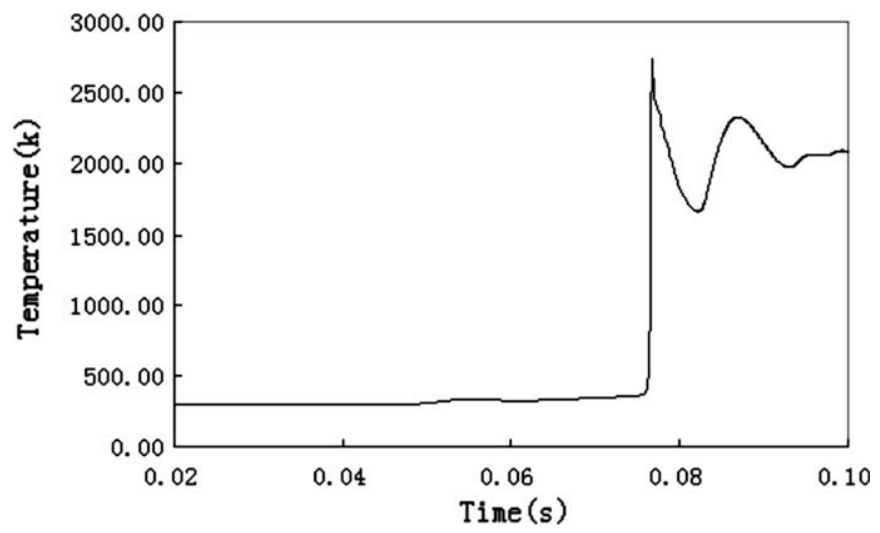

Fig. 8. The temperature history at 6th detector.

pressure peak at the 2nd detector is also displayed in Fig. 7-2. However, comparing Fig. 7-1 with Fig. 7-2, it can be seen that the calculated pressure peak value at the 2 nd detector $(0.4 \mathrm{MPa})$ is much higher than the measured value $(0.15 \mathrm{MPa})$. This is because in the real test tube the wall is not smooth as what adopted for the simulation and the roughness of it is not uniform everywhere. In future work, the test tube should be processed carefully and the friction coefficient of the wall should be accurately measured.

Fig. 8 shows the history of the temperature at the 6th pressure detector. The temperature suddenly increases $0.075 \mathrm{~s}$ after the ignition time and the peak value is over $2500 \mathrm{~K}$. This means that the flame reaches this point at the same time. Comparing Fig. 8 with Fig. 7-2, it can be seen that the temperature peak time is later than the pressure peak time $(0.057 \mathrm{~s})$. This implies that the spread velocity of the flame is lower than the spread velocity of the pressure. Therefore, it can be identified that the combustion remains in the deflagration stage and does not reach the detonation stage. After the flame leaves, the temperature decreases in a fluctuating manner.

The mechanism of the extinguishing of the deflagration can be illustrated as follows. When the pressure signal due to the ignition is registered by the pressure detector placed at the outlet, the valve of the nitrogen tank is opened and $0.4 \mathrm{MPa}$ of nitrogen will be admitted into the tube from the injection position. Nitrogen will dilute the concentrations of both oxygen and $\mathrm{CO}$, and hence the deflagration will be stopped or slowed down. Fig. 9 shows the simulated CO concentration after different times. After injection, the nitrogen immediately flows into the tube and mixes with $\mathrm{CO}$ and air in both the downstream and upstream directions, even at the same time that the CO flame starts to spread. Therefore, the nitrogen has to flow against the upstream CO flame and meanwhile pushes the downstream unburned $\mathrm{CO}$ to the outlet. Hence,

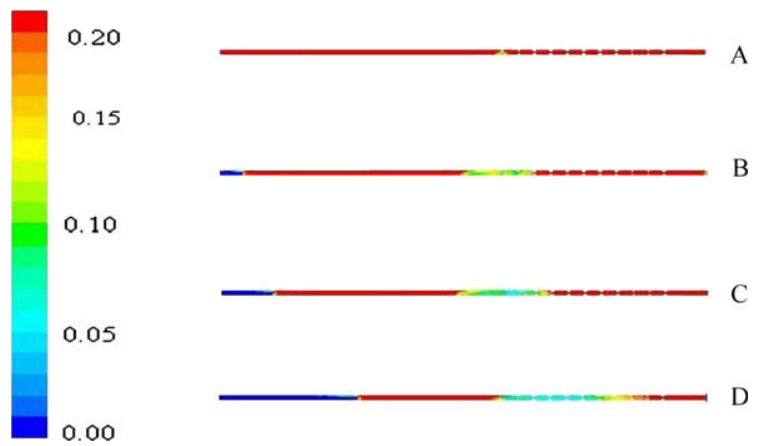

Fig. 9. The CO concentration at different moments. A) As the nitrogen starts to be injected B) $0.05 \mathrm{~s}$ later C) $0.10 \mathrm{~s}$ later D) $0.15 \mathrm{~s}$ later.

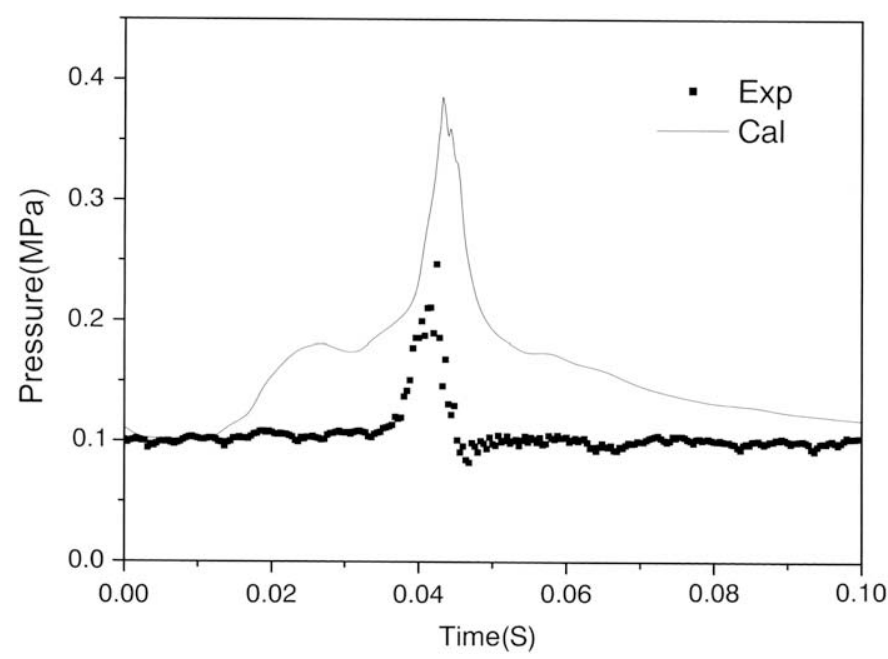

Fig. 10. The pressure history at 6th detector during the extinguishing of deflagration.

a nitrogen block is formed, which becomes broader. Finally, the nitrogen block yields to the high-pressure generated by the $\mathrm{CO}$ flame and flows only in the downstream direction. However, at this moment, a lean CO concentration region is already formed within the nitrogen block. When the upstream CO flame meets the nitrogen block, it will be quenched. It should be pointed out that in real experimental work various values of nitrogen pressure have been tested. If the nitrogen pressure less than 0.4 MPa the deflagration will not be totally quenched. The injected nitrogen of lower pressure will be pushed out from the tube very quickly and the lean CO concentration zone could not be stably formed. Finally there is still weaker but visible flame coming out from the outlet. The cases with injection pressure of nitrogen above $0.4 \mathrm{MPa}$ are not included in this discussion. Detailed tests for the optimization of injection pressure will be put in the future work.

The pressure history at the 6th detector during the extinguishing of the deflagration is demonstrated in Fig. 10. The solid line represents the simulation results and the square symbols represent the experimental results. The simulation results can be compared with the experimental results qualitatively but not quantitatively. The reason for this is that in the real experimental work, the pressure of the injected nitrogen could not always be kept stable at 0.4 MPa. Instead, it varied over a range. Therefore, there is a distinct difference between the simulation and experimental results. Nevertheless, comparing Fig. 10 and Fig. 6, it can still be concluded that the injection of nitrogen slows down the deflagration and that the pressure peak value is reduced by around $50 \%$.

\section{Conclusion}

1. Ignition induces a disturbance in the pressure, which spreads within the semi-opened tube until deflagration starts. It persists from $0.01 \mathrm{~s}$ to $0.06 \mathrm{~s}$.

2. Both experimental and simulation results have shown that deflagration induces a high-pressure peak inside the semiopened tube. The pressure can reach $0.7 \mathrm{MPa}$.

3. The R-S model gives more reasonable estimates than the K-E model in predicting the pressure within the semi-opened tube when deflagration occurs because the latter invariably overestimates the turbulence for an anisotropic turbulent flow.

4. When deflagration happens, if an appropriate amount of nitrogen is injected into the tube, deflagration will be successfully slowed down. The pressure peak value will then be reduced by around $50 \%$. 


\section{Acknowledgement}

This study is sponsored by the National High Technology Research and Development Program ("863"Program) of China (No. 2006AA05Z253)

\section{References}

Chan, C., Moen, I. O., \& Lee, J. H. S. (1983). Influence of confinement on flame acceleration due to repeated obstacles. Combustion and Flame, 49, 27-39.

Fluent 6.3 documentation.

Glassman, I. (1996). Combustion (3rd ed.). San Diego, CA: Academic Press.

Lee, J. H., Knystautas, R., \& Chan, C. K. (1984). Turbulent flame propagation in obstacle-filled tubes. Proceedings of the Combustion Institute, 20, 1663-1672.
Lee, J. H. S., Knystautas, R., \& Freiman, A. (1984). High speed turbulent deflagrations and transition to detonation in $\mathrm{H}_{2}$-air mixtures. Combustion and Flame, 56 , 227-239.

Moen, I. O., Donato, M., Knystautas, R., \& Lee, JH. (1980). Flame acceleration due to turbulence produced by obstacles. Combustion and Flame, 39, 21-32.

Patel, S., Ibrahim, S. S., Yehia, M. A., \& Hargrave, G. K. (2003). Investigation of premixed turbulent combustion in a semi-confined explosion chamber. Experimental Thermal and Fluid Science, 27, 355-361.

Teodorczyk, A., Lee, J. H. S., \& Knystautas, R. (1988). Propagation mechanism of quasi-detonations. Proceedings of the Combustion Institute, 22, 1723-1731.

Yu, L. X., Sun, W. C., \& Wu, C. K. (2002). Flame acceleration and overpressure development in a semi-open tube with repeated obstacles. Proceedings of the Combustion Institute, 29, 321-327.

Yu, L. X., Sun, W. C., \& Wu, C. K. (2002). Influence of obstacle-produced turbulence on development of premixed flames. Science in China (Series E), 45(2), 184-194. 